\title{
Exhausting pants graphs of punctured spheres by finite rigid sets
}

\author{
Rasimate Maungchang \\ School of Science, Walailak University, \\ Nakhon Si Thammarat 80160, Thailand \\ mate105@gmail.com
}

Received 3 June 2017

Revised 23 July 2017

Accepted 24 July 2017

Published 29 November 2017

\begin{abstract}
Let $S_{0, n}$ be an $n$-punctured sphere. For $n \geq 4$, we construct a sequence $\left(\mathcal{X}_{i}\right)_{i \in \mathbb{N}}$ of finite rigid sets in the pants graph $\mathcal{P}\left(S_{0, n}\right)$ such that $\mathcal{X}_{1} \subset \mathcal{X}_{2} \subset \cdots \subset \mathcal{P}\left(S_{0, n}\right)$ and $\bigcup_{i \geq 1} \mathcal{X}_{i}=\mathcal{P}\left(S_{0, n}\right)$.
\end{abstract}

Keywords: Mapping class groups; pants graphs; punctured spheres; rigidity.

Mathematics Subject Classification 2010: 57M99

\section{Introduction}

Let $S=S_{g, n}$ be an orientable surface of genus $g$ with $n$ punctures and let $\operatorname{Mod}^{ \pm}(S)=\pi_{0}(\operatorname{Homeo}(S))$ be the extended mapping class group. Ivanov [6], Korkmaz [7, and Luo [8] proved that, for most surfaces, the curve complexes $\mathcal{C}(S)$ are rigid, that is, $\operatorname{Aut}(\mathcal{C}(S)) \cong \operatorname{Mod}^{ \pm}(S)$. In [2, Aramayona and Leininger proved that curve complexes contain finite rigid sets; meaning a finite subgraph such that every simplicial embedding is a restriction of an element of $\operatorname{Mod}^{ \pm}(S)$. Later in [3, they showed that there exists an exhaustion of the curve complexes by finite rigid sets.

For the pants graphs $\mathcal{P}(S)$, the rigidity property was proved by Margalit 9 using the result of Ivanov, Korkmaz, and Lou. Aramayona [1] extended Margalit's result to prove a stronger form of rigidity, that is, if $S$ and $S^{\prime}$ are surfaces such that the complexity of $S$ is at least 2, then every injective simplicial map $\phi: \mathcal{P}(S) \rightarrow \mathcal{P}\left(S^{\prime}\right)$ is induced by a $\pi_{1}$-injective embedding $f: S \rightarrow S^{\prime}$. In [10, we refined Aramayona's result by showing that the pants graphs of punctured spheres are finitely rigid.

This is an Open Access article published by World Scientific Publishing Company. It is distributed under the terms of the Creative Commons Attribution 4.0 (CC-BY) License. Further distribution of this work is permitted, provided the original work is properly cited. 
In this paper, we modify the tools Aramayona and Leininger built in [3], together with the finite rigid sets we constructed [10, to prove that we can exhaust the pants graphs of punctured spheres by finite rigid sets.

Theorem 1.1. Let $S_{0, n}$ be an $n$-punctured sphere. For $n \geq 4$, there exists a sequence of finite rigid sets $\mathcal{X}_{1} \subset \mathcal{X}_{2} \subset \cdots \subset \mathcal{P}\left(S_{0, n}\right)$ such that $\bigcup_{i \geq 1} \mathcal{X}_{i}=\mathcal{P}\left(S_{0, n}\right)$.

Theorem 1.1 gives us an alternative proof of [9] Theorem 1] for the case of punctured spheres without using the rigidity of curve complexes, as the following corollary states.

Corollary 1.1. Let $S_{0, n}$ be an $n$-punctured sphere. For $n \geq 4$, the natural map $\theta: \operatorname{Mod}^{ \pm}(S) \rightarrow \operatorname{Aut}\left(\mathcal{P}\left(S_{0, n}\right)\right)$ is a surjective homomorphism. If $n=4, \operatorname{ker}(\theta) \cong$ $\mathbb{Z}_{2} \oplus \mathbb{Z}_{2}$. If $n \geq 5, \theta$ is an isomorphism.

Outline of the paper. Section 2 contains the relevant background and definitions. In Sec. 3. we describe the adjustments to the tools Aramayona and Leininger [3] developed to enlarge their rigid sets in the curve complex so we can use them in our setting. We use these tools to prove the main theorem in Sec. 4 .

\section{Background and Definitions}

This section contains necessary definitions and background restricted to punctured spheres, for general settings see [1, 9]. Let $S=S_{0, n}$ be an $n$-punctured sphere. A simple closed curve on $S$ is essential if it does not bound a disk or a once-punctured disk on $S$. Throughout this paper, a curve is a homotopy class of essential simple closed curves on $S$. Given two curves $\gamma$ and $\gamma^{\prime}$, we denote their geometric intersection number by $i\left(\gamma, \gamma^{\prime}\right)$, which is the minimum number of transverse intersection points among the representatives of $\gamma$ and $\gamma^{\prime}$. The two curves are disjoint if $i\left(\gamma, \gamma^{\prime}\right)=0$

A multicurve $Q$ is a set of pairwise distinct, disjoint curves on $S$. For a given multicurve $Q$, the nontrivial piece $(S-Q)_{0}$ of the complement of the curves in $Q$ is the union of the non-thrice-punctured sphere components of the complement. We call a thrice-punctured sphere, a pair of pants.

A pants decomposition $P$ is a maximal multicurve: the complement in $S$ is a disjoint union of pairs of pants. A pants decomposition always contains $n-3$ curves and we call this number the complexity $\kappa(S)$ of $S$. The deficiency of a multicurve $Q$ is the number $\kappa(S)-|Q|$. If $Q$ is a deficiency-1 multicurve then $(S-Q)_{0}$ is homeomorphic to $S_{0,4}$.

Let $P$ and $P^{\prime}$ be pants decompositions of $S$. We say that $P$ and $P^{\prime}$ differ by an elementary move if there are curves $\alpha, \alpha^{\prime}$ on $S$ and a deficiency-1 multicurve $Q$ such that $P=\{\alpha\} \cup Q, P^{\prime}=\left\{\alpha^{\prime}\right\} \cup Q$ and $i\left(\alpha, \alpha^{\prime}\right)=2$; see Fig. 1 for an example of elementary moves. 

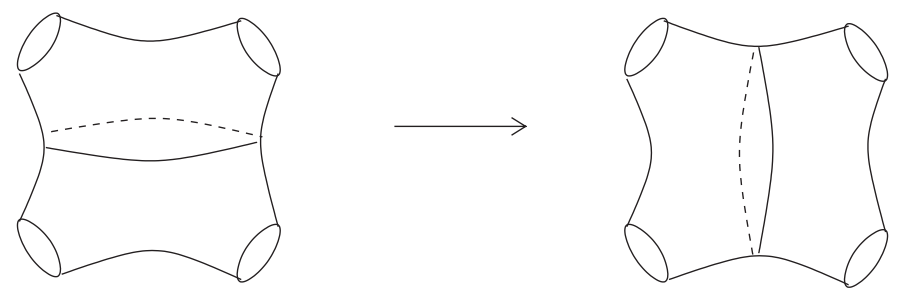

Fig. 1. Example of an elementary move.

The pants graph $\mathcal{P}(S)$ of $S$ is a graph with the set of vertices corresponding to pants decompositions. Two vertices are connected by an edge if the corresponding pants decompositions differ by an elementary move. The pants graph $\mathcal{P}(S)$ is connected and the pants graph $\mathcal{P}\left(S_{0,4}\right)$ of a 4 -punctured sphere is isomorphic to a Farey graph, see [5].

A path in $\mathcal{P}(S)$ is an edge path determined by a sequence of distinct adjacent vertices of $\mathcal{P}(S)$. A cycle in $\mathcal{P}(S)$ is a subgraph homeomorphic to a circle. We call a cycle, a triangle, rectangle, or pentagon if it has 3,4 , or 5 vertices, respectively. Each edge of $\mathcal{P}(S)$ is contained in a unique Farey graph in $\mathcal{P}(S)$, see [9 Lemma 2]. A cycle is called an alternating cycle if any two consecutive edges are in different Farey graphs.

Let $\mathcal{X} \subset \mathcal{P}\left(S_{0, n}\right)$ and $\phi: \mathcal{X} \rightarrow \mathcal{P}\left(S_{0, m}\right)$ be an injective simplicial map. We say that a $\pi_{1}$-injective embedding $f: S_{0, n} \rightarrow S_{0, m}$ induces $\phi$ if there is a deficiency$(n-3)$ multicurve $Q$ on $S_{0, m}$ such that $f\left(S_{0, n}\right)=\left(S_{0, m}-Q\right)_{0}$ and the simplicial map

$$
f^{Q}: \mathcal{P}\left(S_{0, n}\right) \rightarrow \mathcal{P}\left(S_{0, m}\right),
$$

defined by $f^{Q}(u)=f(u) \cup Q$ satisfies $f^{Q}(u)=\phi(u)$ for any vertex $u \in \mathcal{X}$.

Definition 2.1. For $n \geq 4$, we say that $\mathcal{X} \subset \mathcal{P}\left(S_{0, n}\right)$ is rigid if for any punctured sphere $S_{0, m}$ and any injective simplicial map

$$
\phi: \mathcal{X} \rightarrow \mathcal{P}\left(S_{0, m}\right),
$$

there exists a $\pi_{1}$-injective embedding $f: S_{0, n} \rightarrow S_{0, m}$ that induces $\phi$, unique up to the pointwise stabilizer of $\mathcal{X}$ in $\operatorname{Mod}^{ \pm}\left(S_{0, n}\right)$.

The following theorem is a refinement of Aramayona's result [1] that we proved in [10.

Theorem 2.1. For $n \geq 4$, there exists a finite rigid subgraph $X_{n} \subset \mathcal{P}\left(S_{0, n}\right)$. 


\section{Tools for Enlarging Rigid Sets}

This section contains the definitions and theorems Aramayona and Leininger [3] developed to enlarge their rigid sets in curve complexes. We make some necessary adjustments to them in order to enlarge rigid sets in pants graphs.

Definition 3.1. Let $n \geq 5$. A set $\mathcal{X} \subset \mathcal{P}\left(S_{0, n}\right)$ is said to be weakly rigid if whenever $f_{1}, f_{2}: S_{0, n} \rightarrow S_{0, m}$ are $\pi_{1}$-injective embeddings satisfy

$$
\left.f_{1}^{Q_{1}}\right|_{\mathcal{X}}=\left.f_{2}^{Q_{2}}\right|_{\mathcal{X}}
$$

for some deficiency- $(n-3)$ multicurves $Q_{1}$ and $Q_{2}$ on $S_{0, m}$, then

$$
Q_{1}=Q_{2} \quad \text { and } \quad f_{1}=f_{2}
$$

up to isotopy.

It is easy to see from the definition that a superset of a weakly rigid set is also weakly rigid.

Lemma 3.1. For $n \geq 5$, let $\mathcal{X}_{1}, \mathcal{X}_{2} \subset \mathcal{P}\left(S_{0, n}\right)$ be rigid sets. If $\mathcal{X}_{1} \cap \mathcal{X}_{2}$ is weakly rigid then $\mathcal{X}_{1} \cup \mathcal{X}_{2}$ is rigid.

Proof. Let $\phi: \mathcal{X}_{1} \cup \mathcal{X}_{2} \rightarrow \mathcal{P}\left(S_{0, m}\right)$ be an injective simplicial map. Since $\mathcal{X}_{i}$ is rigid, there exist a $\pi_{1}$-injective embedding $f_{i}: S_{0, n} \rightarrow S_{0, m}$ and a deficiency- $(n-3)$ multicurve $Q_{i}$ such that $\left.f_{i}^{Q_{i}}\right|_{\mathcal{X}_{i}}=\left.\phi\right|_{\mathcal{X}_{i}}$. Hence $\left.f_{1}^{Q_{1}}\right|_{\mathcal{X}_{1} \cap \mathcal{X}_{2}}=\left.\phi\right|_{\mathcal{X}_{1} \cap \mathcal{X}_{2}}=\left.f_{2}^{Q_{2}}\right|_{\mathcal{X}_{1} \cap \mathcal{X}_{2}}$. The weak rigidity of $\mathcal{X}_{1} \cap \mathcal{X}_{2}$ implies that $Q_{1}=Q_{2}=Q$ and $f_{1}=f_{2}=f$. Therefore, $f$ is a $\pi_{1}$-injective embedding such that $\left.f^{Q}\right|_{\mathcal{X}_{1} \cup \mathcal{X}_{2}}=\phi$ which implies the rigidity of $\mathcal{X}_{1} \cup \mathcal{X}_{2}$.

Let $T_{\alpha}^{\frac{1}{2}} \in \operatorname{Mod}\left(S_{0, n}\right)$ be a half-twist around a curve $\alpha$ on $S_{0, n}$. In this paper, we will not distinguish between homeomorphisms and their homotopy classes. The following proposition is the key to enlarge rigid sets.

Proposition 3.2. For $n \geq 5$, let $\mathcal{X} \subset \mathcal{P}\left(S_{0, n}\right)$ be a finite rigid set such that $\operatorname{Mod}\left(S_{0, n}\right) \cdot \mathcal{X}=\mathcal{P}\left(S_{0, n}\right)$. Suppose there exists a finite subset $C$ of curves on $S_{0, n}$ such that:

(1) The set $\left\{T_{\alpha}^{ \pm \frac{1}{2}} \mid \alpha \in C\right\}$ generates $\operatorname{Mod}\left(S_{0, n}\right)$;

(2) $\mathcal{X} \cap T_{\alpha}^{i}(\mathcal{X})$ is weakly rigid, for all $\alpha \in C$, and $i \in\left\{-\frac{1}{2}, \frac{1}{2}\right\}$.

Then there exists a sequence $\mathcal{X}=\mathcal{X}_{1} \subset \mathcal{X}_{2} \subset \cdots \subset \mathcal{X}_{n} \subset \cdots$ such that each $\mathcal{X}_{i}$ is a finite rigid set, and

$$
\bigcup_{i \in \mathbb{N}} \mathcal{X}_{i}=\mathcal{P}\left(S_{0, n}\right)
$$


Proof. Since $\mathcal{X}$ is rigid and a half-twist is a homeomorphism, $T_{\alpha}^{i}(\mathcal{X})$ is rigid for all $\alpha \in C$, and $i \in\left\{-\frac{1}{2}, \frac{1}{2}\right\}$. Given $\alpha, \beta \in C$ and $i, j \in\left\{-\frac{1}{2}, \frac{1}{2}\right\}$. By assumption (2) and by applying Lemma 3.1, we see that $\mathcal{X} \cup T_{\alpha}^{i}(\mathcal{X})$ is rigid. Recall that a superset of a weakly rigid set is also weakly rigid. Hence $\left(\mathcal{X} \cup T_{\alpha}^{i}(\mathcal{X})\right) \cap T_{\beta}^{j}(\mathcal{X})$, which contains $\mathcal{X} \cap T_{\beta}^{j}(\mathcal{X})$, is weakly rigid. Applying Lemma 3.1 we see that $\mathcal{X} \cup T_{\alpha}^{i}(\mathcal{X}) \cup T_{\beta}^{j}(\mathcal{X})$ is weakly rigid. By repeating above arguments, the set $\mathcal{X}_{2}:=\mathcal{X} \cup \cup_{\alpha \in C} T_{\alpha}^{ \pm \frac{1}{2}}(\mathcal{X})$ is rigid. We define

$$
\mathcal{X}_{n+1}:=\mathcal{X}_{n} \cup \bigcup_{\alpha \in C} T_{\alpha}^{ \pm \frac{1}{2}}\left(\mathcal{X}_{n}\right),
$$

for $n \geq 2$. Since the weakly rigid set $\mathcal{X} \cap T_{\alpha}^{i}(\mathcal{X})$ is a subset of $\mathcal{X}_{n} \cap T_{\alpha}^{i}\left(\mathcal{X}_{n}\right)$, $\mathcal{X}_{n} \cap T_{\alpha}^{i}\left(\mathcal{X}_{n}\right)$ is weakly rigid. Again, by applying Lemma 3.1 inductively and use induction, we conclude that $\mathcal{X}_{n}$ is rigid for all $n$. Then the first claim is proved.

Finally, since $\left\{T_{\alpha}^{ \pm \frac{1}{2}} \mid \alpha \in C\right\}$ generates $\operatorname{Mod}\left(S_{0, n}\right)$ and $\operatorname{Mod}\left(S_{0, n}\right) \cdot \mathcal{X}=\mathcal{P}\left(S_{0, n}\right)$,

$$
\bigcup_{i \in \mathbb{N}} \mathcal{X}_{i}=\mathcal{P}\left(S_{0, n}\right)
$$

\section{The Proof of the Main Theorem}

We note that for $n \leq 3$, the pants graphs $\mathcal{P}\left(S_{0,3}\right)$ is empty. We give a separate proof for $n=4$, which can also be found in [3] Sec. 4.1], as follows.

Proof of Theorem 1.1 for $\boldsymbol{S}=\boldsymbol{S}_{\mathbf{0 , 4}}$. The pants graph of $S_{0,4}$ is isomorphic to the Farey graph. Any triangle in $S_{0,4}$ is rigid as proved in [10]. Then we let $\mathcal{X}_{1}$ to be a triangle. Each edge in a pants graph of any punctured sphere is contained in exactly two triangles which are both in the same Farey graph. Then we can define $\mathcal{X}_{n+1}$ inductively; let $\mathcal{X}_{n+1}$ be an enlargement of $\mathcal{X}_{n}$ obtained by attaching one more triangle to each edge of $\mathcal{X}_{n}$ contained in only one triangle. Hence $\mathcal{X}_{n+1}$ is rigid for all $n \geq 1$, and by the construction, $\bigcup_{i \in \mathbb{N}}\left(\mathcal{X}_{i}\right)=\mathcal{P}\left(S_{0,4}\right)$. We conclude that sequence $\left(\mathcal{X}_{n}\right)_{n \in \mathbb{N}}$ is an exhaustion of $\mathcal{P}\left(S_{0,4}\right)$.

For $n \geq 5$, we begin by recalling the construction of finite rigid sets $X_{n}$ in [10]. First, we construct $S_{0, n}$ with a set of curves, then define $X_{5}$, and finally, define $X_{n}$ for $n \geq 6$.

Consider a regular $n$-gon with the $n$ vertices removed and label the sides as $1,2, \ldots, n$, cyclically. For each non-adjacent pair of sides of the $n$-gon, draw a straight line segment to connect the two sides. Then double the $n$-gon to obtain $S_{0, n}$ and a set of curves $\Gamma_{n}$, see Fig. 2 for the case of $S_{0,8}$ and Fig. 3 for the case of $S_{0,5}$. Let $a_{i, j} \in \Gamma_{n}$ be the curve connecting the $i$ th side to the $j$ th side of $S_{n}$. We call $a_{i, j}$ such that $i-j \equiv \pm 2 \bmod n$, a chain curve. Compare to [2] Sec. 3].

Let $Z_{n}$ be a subgraph of $\mathcal{P}\left(S_{0, n}\right)$ induced by the set of vertices corresponding to pants decompositions consisting of curves from $\Gamma_{n}$. 


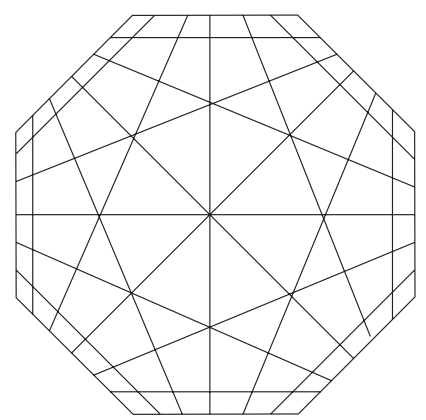

Fig. 2. $S_{0,8}$ and the set of simple closed curves $\Gamma_{8}$.
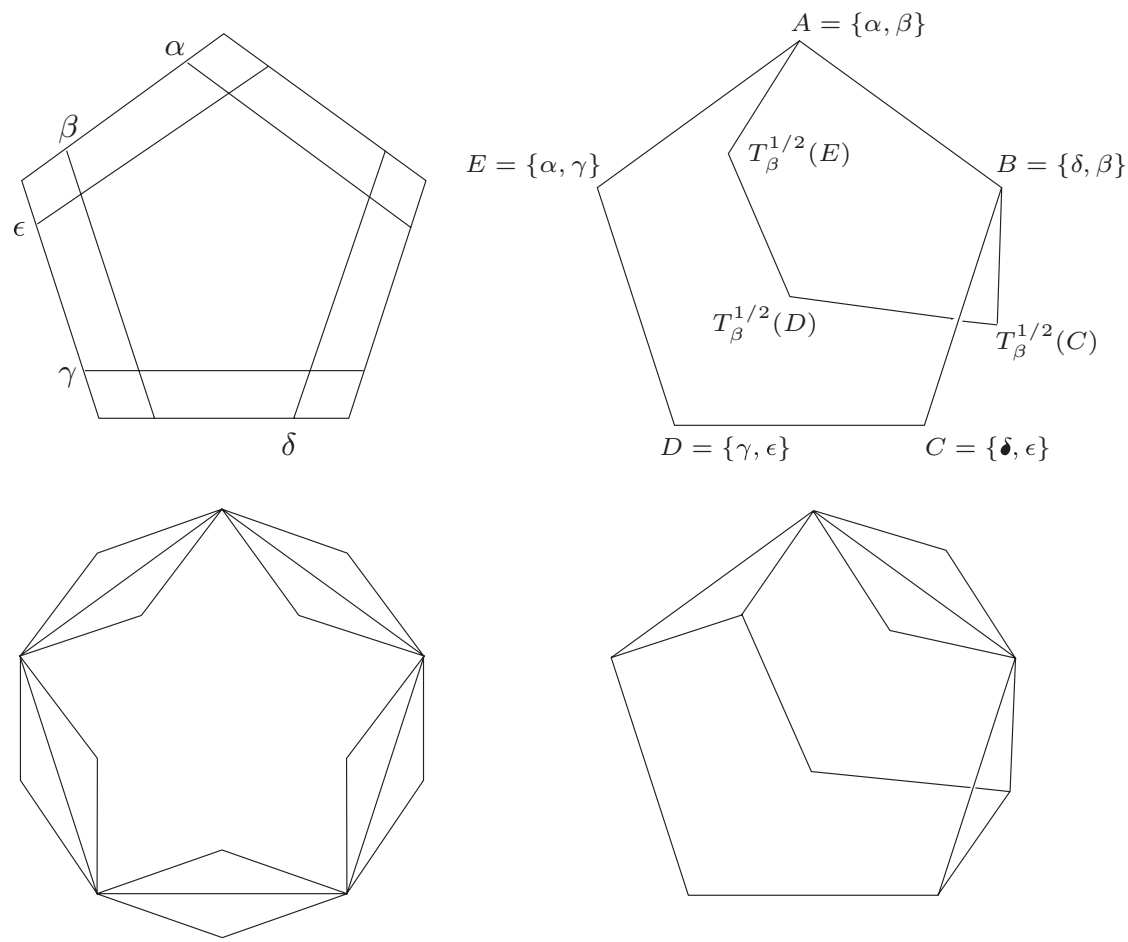

Fig. 3. (Top left) $S_{0,5}$ and curves in $\Gamma_{5}$, (top right) $Z_{5} \cup T_{\beta}^{\frac{1}{2}}\left(Z_{5}\right.$ ), (bottom left) $Z_{5}$ together with the 10 triangles, and (bottom right) $X_{5} \cap T_{\alpha}^{\frac{1}{2}}\left(X_{5}\right)$.

For $\mathcal{P}\left(S_{0,5}\right)$, we defined

$$
X_{5}=Z_{5} \cup \bigcup_{c \in \Gamma_{5}} T_{c}^{ \pm \frac{1}{2}}\left(Z_{5}\right)
$$

where $T_{c}^{\frac{1}{2}}$ is a simplicial map on $\mathcal{P}\left(S_{0,5}\right)$ induced by the half-twist around the curve $c$. 
See Fig. 3 for a partial figure of $X_{5}$. The subgraph $X_{5}$ consists of the alternating pentagon $Z_{5}$ and 10 of its images under the twists. Those 10 images form 10 triangles attached to $Z_{5}$. In [10], we proved that $X_{5}$ is rigid.

For $n \geq 6$, we construct $X_{n}$ as follows. Let $W \subset \Gamma_{n}$ be a deficiency-2 multicurve such that $\left(S_{0, n}-W\right)_{0} \cong S_{0,5}$. Let $\Gamma_{5}^{W}=\left\{\alpha \in \Gamma_{n} \mid \alpha\right.$ is disjoint from all curves in $\left.W\right\}$. There is a natural homeomorphism $h: S_{0,5} \rightarrow\left(S_{0, n}-W\right)_{0}$ such that $h\left(\Gamma_{5}\right)=\Gamma_{5}^{W}$, see [10, Lemma 3.1]. Let

$$
X_{5}^{W}=h^{W}\left(X_{5}\right)=\left\{h(u) \cup W \mid u \in X_{5}\right\},
$$

where $h^{W}: P\left(S_{0,5}\right) \rightarrow P\left(S_{0, n}\right)$ is the induced map of $h$ defined by $h^{W}(u)=$ $h(u) \cup W$. Then $X_{5}^{W} \cong X_{5}$. Finally, we let

$$
X_{n}=Z_{n} \cup \bigcup_{W} X_{5}^{W},
$$

where the union is taken over all deficiency-2 multicurves in $\Gamma_{n}$ with a 5 -punctured sphere component. In [10, we proved that $X_{n}$ is rigid.

In the light of Proposition 3.2 we need the following lemmas to prove the main theorem for $n \geq 5$.

Lemma 4.1. $\operatorname{Mod}\left(S_{0, n}\right) \cdot X_{n}=\mathcal{P}\left(S_{0, n}\right)$

Proof. In the first part of this proof, we will show that, for a given vertex $P$ in $\mathcal{P}\left(S_{0, n}\right)$, there exists a vertex $P^{\prime}$ in $X_{n}$ and $f \in \operatorname{Mod}\left(S_{0, n}\right)$ such that $f\left(P^{\prime}\right)=P$. To do this, we obtain a pants decomposition $P^{\prime}$ from a dual graph of the pants decomposition $P$. For the second part, we will show that there is a homeomorphism that sends a given edge in $\mathcal{P}\left(S_{0, n}\right)$ to an edge in $Z_{n} \subset X_{n}$.

Let $P$ be a vertex of $\mathcal{P}\left(S_{0, n}\right)$. Recall that we consider $S_{0, n}$ as a double of a regular $n$-gon. Consider $P$ as a pants decomposition on $S_{0, n}$. The following construction of a dual graph of $P$ was given in [5]. For each pair of pants component of $\left(S_{0, n}-P\right)$, we mark a vertex on the interior of the component. We also mark the $n$ punctures as $n$ vertices. Two vertices are connected by an edge if (1) they are vertices on the interior of two pants components which have a common boundary, or (2) one of the vertices is on the interior of a pair of pants component and another vertex is a puncture of the same component. The result is a tree with $2 n-2$ vertices; all puncture-vertices have degree 1 , while the rest of the vertices has degree 3 , see Fig. 4.

Since a tree is planar, we can redraw this tree on the plane inside a regular $n$-gon so that all $n$ puncture-vertices are the $n$ vertices of the $n$-gon. We reconstruct a pants decomposition consisting of curves in $\Gamma_{n}$ by drawing a curve connecting two sides of the regular $n$-gon whenever this curve can cross exactly one edge of the tree and both endpoints of this edge are not puncture-vertices. Double the regular $n$-gon. We now have a pants decomposition $P^{\prime}$ consisting of curves in $\Gamma_{n}$, i.e. $P^{\prime}$ is a vertex in $Z_{n} \subset X_{n}$. 


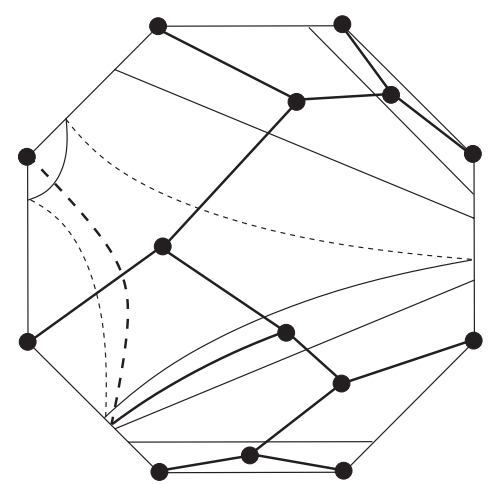

Fig. 4. Example of a pants decomposition of $S_{0,8}$ and its dual graph shown in thick edges.

The above construction of $P^{\prime}$ from $P$ gives a one-to-one correspondence between the pants components $S_{0, n}-P$ and the pants components $S_{0, n}-P^{\prime}$. This correspondence describes a homeomorphism $f$ such that $f\left(P^{\prime}\right)=P$, as desired.

Next, we show that if $P_{1}$ and $P_{2}$ are adjacent vertices in $\mathcal{P}\left(S_{0, n}\right)$, then after applying some homeomorphisms on $S_{0, n}$ to $P_{1}$ and $P_{2}$, we get two vertices that are adjacent in $Z_{n}$.

Given adjacent vertices $P_{1}$ and $P_{2}$ in $\mathcal{P}\left(S_{0, n}\right)$, then there exist curves $u_{1}, u_{2}$ on $S_{0, n}$ and a deficiency-1 multicurve $Q$ such that $P_{1}=\left\{u_{1}\right\} \cup Q$ and $P_{2}=\left\{u_{2}\right\} \cup Q$.



Fig. 5. Example of an edge $\left\{f\left(P_{1}\right), f\left(P_{2}\right)\right\}$ and its images after composing with a power of full twist around the curve $f\left(u_{1}\right)$ and a half twist around the same curve. 
By the first part of the proof, there is $f \in \operatorname{Mod}\left(S_{0, n}\right)$ such that $f\left(P_{1}\right)$ is a vertex in $Z_{n}$. If $f\left(P_{2}\right)$ is also in $Z_{n}$, then we are done.

Suppose $f\left(P_{2}\right)$ is not in $Z_{n}$. Use Fig. [5 as a reference for the rest of the proof. We note that $f(Q) \subset \Gamma_{n}$ and it has deficiency-1. The nontrivial component $\left(S_{0, n}-\right.$ $f(Q))_{0} \cong S_{0,4}$ contains exactly two curves in $\Gamma_{n}$; one curve is $f\left(u_{1}\right)$ and we call the other curve $\alpha$. Then $i\left(f\left(u_{2}\right), \alpha\right)=2 n$ for some $n \in \mathbb{N}$. Applying one full twist around $f\left(u_{1}\right)$ in an appropriate direction reduces the intersection number by 4 . Observe that $f\left(P_{1}\right)$ is invariant under this full twist. So we can choose a new $f$ (by composing the old one with some power of full twists) and assume that $i\left(f\left(u_{2}\right), \alpha\right)=0$ or $i\left(f\left(u_{2}\right), \alpha\right)=2$. If $i\left(f\left(u_{2}\right), \alpha\right)=0$, then $f\left(u_{2}\right)=\alpha$ and we are done.

Suppose $i\left(f\left(u_{2}\right), \alpha\right)=2$. We compose $f$ by an appropriate half twist $T$ around $f\left(u_{1}\right)$ : here a half-twist in $f\left(u_{1}\right)$ is a homeomorphism on $S_{0, n}$, whose square is the Dehn twist in $f\left(u_{1}\right)$, although we note that it does not necessary to restrict a homeomorphism of $\left(S_{0, n}-f(Q)\right)_{0} \cong S_{0,4}$. We choose the half-twist that essentially "flips over" half of the $n$-gon, cut along $f\left(u_{1}\right)$; see Figs. 5 and 6 Then $T \circ f\left(u_{2}\right)=\alpha$ and the edge $\left\{T \circ f\left(P_{1}\right), T \circ f\left(P_{2}\right)\right\}$ is in $Z_{n}$ as desired.

Let $\alpha$ be a curve on $S_{0, n}$. We define $\mathcal{P}_{\alpha}\left(S_{0, n}\right)$ to be a subgraph of $\mathcal{P}\left(S_{0, n}\right)$ induced by vertices corresponding to pants decompositions containing $\alpha$.

The following lemma is proved in [10] and we use this lemma to prove Lemma 4.3
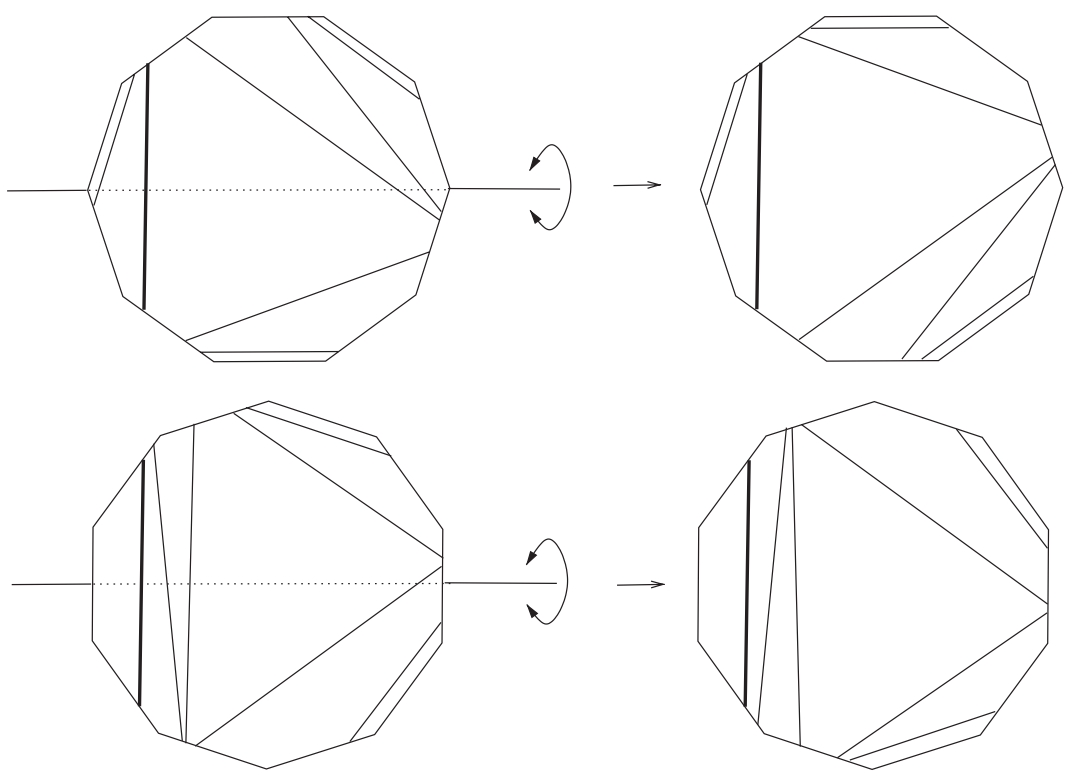

Fig. 6. Examples of half-twist around the thick curves. Two pants decompositions in $Z_{10}$ and $Z_{11}$ are given to help visualize the homeomorphisms. Note that after a half twisting, we get a new pants decomposition that is still in $Z_{10}$ or $Z_{11}$. 
Lemma 4.2. For $n \geq 6$, let $\alpha$ be a chain curve on $S_{0, n}$ and let $X_{n-1}^{\alpha}=X_{n} \cap$ $\mathcal{P}_{\alpha}\left(S_{0, n}\right)$.

Then $X_{n-1}^{\alpha} \cong X_{n-1}$. Moreover, this isomorphism is induced by $h: S_{0, n-1} \rightarrow$ $\left(S_{0, n}-\alpha\right)_{0}$ as $h^{\alpha}(v)=h(v) \cup\{\alpha\} \in X_{n-1}^{\alpha}$.

Lemma 4.3. $X_{n} \cap T_{\alpha}^{i}\left(X_{n}\right)$ is weakly rigid, for $i \in\left\{-\frac{1}{2}, \frac{1}{2}\right\}$ and for all chain curves $\alpha$ in $S_{0, n}$.

Proof. Let $\alpha$ be a chain curve and $i \in\left\{-\frac{1}{2}, \frac{1}{2}\right\}$. Suppose $f_{1}, f_{2}: S_{0, n} \rightarrow S_{0, m}$ are $\pi_{1}$-injective embeddings such that

$$
\left.f_{1}^{Q_{1}}\right|_{X_{n} \cap T_{\alpha}^{i}\left(X_{n}\right)}=\left.f_{2}^{Q_{2}}\right|_{X_{n} \cap T_{\alpha}^{i}\left(X_{n}\right)},
$$

for some deficiency- $(n-3)$ multicurves $Q_{1}$ and $Q_{2}$ on $S_{0, m}$.

We first prove the case of $n=5$. Recall the definition of $X_{5}$ and the definition of an alternating cycle. By a direct calculation, we see that $X_{5} \cap T_{\alpha}^{i}\left(X_{5}\right)$ consists of two alternating pentagons which are $Z_{5}=T_{\alpha}^{i}\left(T_{\alpha}^{-i}\left(Z_{5}\right)\right)$ and $T_{\alpha}^{i}\left(Z_{5}\right)$. They share an edge together with four triangles as shown in Fig. 3 . Since $Z_{5}$ is an alternating pentagon and $\left.f_{1}^{Q_{1}}\right|_{Z_{5}}=\left.f_{2}^{Q_{2}}\right|_{Z_{5}}$, [8, Lemma 4.2] implies that $Q_{1}=Q_{2}$ and

$$
f_{1}=f_{2} \text { or } f_{1}=f_{2} \circ e,
$$

where $e: S_{0,5} \rightarrow S_{0,5}$ is the involution exchanging the two pentagons (as we consider $S_{5}$ as a double of a pentagon). The map $e$ induces a simplicial map on $\mathcal{P}\left(S_{0,5}\right)$ that fixes $Z_{5}$ and exchanges two triangles on each side of $Z_{5}$. But $f_{1}$ and $f_{2}$ also agree on the four triangles attached to $Z_{5}$ so $f_{1}=f_{2}$. Hence the case of $n=5$ is proved.

Let $n \geq 6$ and let $\alpha$ be any chain curve. By Lemma 4.2 a subgraph $X_{n-1}^{\alpha}=$ $X_{n} \cap \mathcal{P}_{\alpha}\left(S_{0, n}\right) \cong X_{n-1}$. Since each vertex of $X_{n-1}^{\alpha}$ contains $\alpha, T_{\alpha}^{i}\left(X_{n-1}^{\alpha}\right)=X_{n-1}^{\alpha}$. Hence $X_{n} \cap T_{\alpha}^{i}\left(X_{n}\right)$ contains $X_{n-1}^{\alpha} \cong X_{n-1}$. Consider the restrictions of $f_{1}$ and $f_{2}$ on the subsurface $\left(S_{0, n}-\{\alpha\}\right)_{0}$. Since $X_{n-1}$ is rigid, so is $X_{n-1}^{\alpha}$, and the uniqueness part of Definition 2.1 implies that $f_{1}$ agrees with $f_{2}$ on $\left(S_{0, n}-\{\alpha\}\right)_{0}$ and $Q_{1} \cup\left\{f_{1}(\alpha)\right\}=$ $Q_{2} \cup\left\{f_{1}(\alpha)\right\}$.

We can see that $X_{n-1}^{\alpha}$ is a proper subgraph of $X_{n} \cap T_{\alpha}^{i}\left(X_{n}\right)$. For example, choose a vertex $P$ in $Z_{n} \cap \mathcal{P}_{\alpha}\left(S_{0, n}\right) \subset X_{n-1}^{\alpha}$. Then change $P$ to $P^{\prime}$ by the elementary move which replaces $\alpha$ by the other curve $\alpha^{\prime}$ in $\Gamma_{n}$. The vertex $T_{\alpha}^{i}\left(P^{\prime}\right)$ is adjacent to $P$ and it is a vertex in $X_{n} \cap T_{\alpha}^{i}\left(X_{n}\right)$. Hence $f_{1}$ and $f_{2}$ agree on $T_{\alpha}^{i}\left(P^{\prime}\right)$. Since $Q_{1}$ and $Q_{2}$ are the intersections of all vertices in $f_{1}\left(X_{n} \cap T_{\alpha}^{i}\left(X_{n}\right)\right)$ and $f_{2}\left(X_{n} \cap T_{\alpha}^{i}\left(X_{n}\right)\right)$, respectively, and $\alpha \notin T_{\alpha}^{i}\left(P^{\prime}\right)$, it follows $f_{1}(\alpha)=f_{2}(\alpha)$ is not in the intersection. Therefore, $Q_{1}=Q_{2}$ and $f_{1}=f_{2}$.

Proof of Theorem 1.1 for $\boldsymbol{S}_{\mathbf{0}, \boldsymbol{n}}, \boldsymbol{n} \geq \mathbf{5}$. We are ready to prove the main theorem for $n \geq 5$. We will check that all conditions in Proposition 3.2 are satisfied.

Let $\mathcal{X}=X_{n}$. Lemma 4.1 states that $\operatorname{Mod}\left(S_{0, n}\right) \cdot \mathcal{X}=\mathcal{P}\left(S_{0, n}\right)$. The set

$$
C=\left\{T^{ \pm \frac{1}{2}}(\alpha) \mid \alpha \text { a chain curve }\right\}
$$

generates $\operatorname{Mod}\left(S_{0, n}\right)$, see [4, Corollary 4.15], hence the condition (1) in Proposition 3.2 is satisfied. By Lemma 4.3, $X_{n} \cap T_{\alpha}^{i}\left(X_{n}\right)$ is weakly rigid, for $i \in\left\{-\frac{1}{2}, \frac{1}{2}\right\}$ 
and for all chain curves $\alpha$ in $S_{0, n}$, hence the condition (2) in Proposition 3.2 is satisfied. Therefore, Proposition 3.2 gives us a sequence of finite rigid set $\mathcal{X}=\mathcal{X}_{1} \subset \mathcal{X}_{2} \subset \cdots \subset \mathcal{X}_{m} \subset \cdots$ such that $\bigcup_{i \in \mathbb{N}} \mathcal{X}_{i}=\mathcal{P}\left(S_{0, n}\right)$, as desired.

\section{Acknowledgments}

The author would like to thank Christopher J. Leininger for his guidance, useful conversations, and suggestions. In addition, the author would like to thank the referees for their thorough review and valuable comments.

\section{References}

[1] J. Aramayona, Simplicial embeddings between pants graphs, Geom. Dedicata 144(1) (2010) 115-128.

[2] J. Aramayona and C. J. Leininger, Finite rigid sets in curve complexes, J. Topol. Anal. 5(2) (2013) 183-203.

[3] J. Aramayona and C. J. Leininger, Exhausting curve complexes by finite rigid sets, Pacific J. Math. 282(2) (2016) 257-283.

[4] B. Farb and D. Margalit, A Primer on Mapping Class Groups (PMS-49) (Princeton University Press, 2011).

[5] A. Hatcher and W. Thurston, A presentation for the mapping class group of a closed orientable surface, Topology 19(3) (1980) 221-237.

[6] N. V. Ivanov, Automorphism of complexes of curves and of teichmüller spaces, Int. Math. Res. Not. 1997(14) (1997) 651-666.

[7] M. Korkmaz, Automorphisms of complexes of curves on punctured spheres and on punctured tori, Topology Appl. 95(2) (1999) 85-111.

[8] F. Luo, Automorphisms of the complex of curves, Topology 39(2) (2000) 283-298.

[9] D. Margalit, Automorphisms of the pants complex, Duke Math. J. 121(3) (2004) 457-479.

[10] R. Maungchang, Finite rigid subgraphs of the pants graphs of punctured spheres, preprint (2013), arXiv:1303.3873v2. 